\title{
Editorial
}

\section{Are transient environmental agents involved in the aetiology of primary biliary cirrhosis?}

\author{
Richard J.Q. McNally*
}

Recently a space-time clustering analysis of cases of primary biliary cirrhosis (PBC) was carried out, using population-based data from a defined geographical region of northeast England. Space-time clustering analysis is a specialised descriptive statistical technique and is observed when excess cases are found in highly localised geographical areas for very limited periods of time. The analyses revealed the presence of highly statistically significant space-time clustering which could not be attributed to an artefactual occurrence. This finding was interpreted as providing supportive evidence for the involvement of transient environmental agents in aetiology [1].

This Special Issue of Disease Markers is dedicated to PBC and specifically the role that transient environmental factors may play in aetiology. Current knowledge is reviewed by seven selected invited experts (and associates) in the field.

The aetiology of PBC is far from clear. In this Special Issue of Disease Markers, the role of transient environmental agents is reviewed by the seven invited contributors. They each provide their own perspective to the ongoing debate. The possibility that the putative transient agent might be an infection is considered by some of the reviewers. Epidemiological studies have indicated that infections may either trigger PBC or lead to progression. Reviewers also examine the putative role of other transient environmental factors, including chemical exposures, contaminated drinking water and lifestyle factors.

*Corresponding author: Dr. Richard J.Q. McNally, Institute of Health and Society, Newcastle University, Sir James Spence Institute, Royal Victoria Infirmary, Queen Victoria Road, Newcastle upon Tyne NE1 4LP, England, United Kingdom. Tel.: +44 191202 3029; Fax: +44 191202 3060; E-mail: Richard.McNally@ncl.ac.uk.
In the first review, Ortega-Hernandez and colleagues examine the evidence for an infectious involvement in aetiology by revisiting all reports that suggest that infections may be implicated in the autoimmune pathogenesis of PBC (Ortega-Hernandez O-D, Levin $N-A$ and Shoenfeld $Y$. Infectious agents in the pathogenesis of primary biliary cirrhosis). They also consider the immune molecular mimicry mechanisms that may be involved with the autoimmune process in PBC. Since there is a 50-100 fold higher relative risk in first-degree relatives and a high concordance rate amongst identical twins, aetiology must involve both genetic and environmental factors. Candidate infectious agents included Escherichia coli, Chlamydia pneumoniae, Mycobacterium gordonae and Novosphingobium aromaticivorans. They conclude that the exact pathogenesis is complex and the exact mechanism implicated in the induction of anti-mitochondrial responses is not well understood.

Selmi and colleagues, in the second review, consider the evidence concerning both infectious agents and chemical xenobiotics in the aetiology of PBC (Selmi $C$, De Santis M, Cavaciocchi $F$ and Gershwin ME. Infectious agents and xenobiotics in the etiology of primary biliary cirrhosis). They also acknowledge that both genetic and environmental exposures must be involved. They found that whilst specific agents could not be identified, the overall body of evidence supports complex mechanisms involving roles for multiple infectious agents and/or xenobiotics. However, they point out that epigenetic changes might also be responsible, which has been proposed for other autoimmune diseases. They stress that there are difficulties in understanding the role of possible environmental agents because of the long latency period from exposure to 
disease onset and also due to possible confounding factors.

In contrast to first two reviews, Bogdanos and colleagues consider a specific agent, E. coli, and, in the third review, focus on the evidence linking this particular bacterium with the disease's development (Bogdanos DP, Baum H, Vergani D and Burroughs AK. E. coli infection as a marker of primary biliary cirrhosis). There is a large body of epidemiological and immunological evidence that supports a role for this particular agent in the aetiology of PBC. However, although there are highly plausible mechanisms they have still not been proven to be causal and further research on $E$. coli is required.

Kumagi and colleagues, in the fourth review, critically assess the case for and against the involvement of infections in pathogenesis (Kumagi T, Abe M, Ikeda Y and Hiasa Y. Infection as a risk factor in the pathogenesis of primary biliary cirrhosis: pros and cons). They also reviewed a number of environmental agents that are likely to be involved including cigarette smoke, nail polish, and hair dyes. They concluded that infections, especially bacterial agents such as $E$. coli, are likely to be a major risk factor. This is because such agents in autoimmune diseases express antigens that result in molecular mimicry and xenobiotics that contribute to tolerance being broken.

Other environmental risk factors have been postulated as being involved with the pathogenesis of PBC. Dronamraju and colleagues, in the fifth review, consider environmental risk factors and possible explanations for spatial clusters (Dronamraju D, Odin J and Bach N. Primary Biliary Cirrhosis: Environmental Risk Factors). Factors implicated included water, sunlight, radiation and toxic waste. Additionally, they also consider xenobiotics, including benzene, polychlorethylene, 6-bromohexonate, 2-octynoic acid, halothane and other halogenated hydrocarbons, and microbial exposures, including E. coli, N. aromaticivorans, Human beta retrovirus, Chlamydia, $P$. acnes, Lactobacillus and Mycobacterium. They concluded that aetiology, although still far from clear, must be complex and multifactorial.

In the sixth review, Kouroumalis also considers environmental agents in general, including lifestyle factors such as smoking and alcohol consumption, infections, reproductive factors, chemical exposures and radiation (Kouroumalis E. Environmental agents involved in the cause of Primary Biliary Cirrhosis). Patients with PBC reported significantly higher rates of tonsillectomy and appendectomy. In one case-control study, no history of pregnancy was associated with protection against $\mathrm{PBC}$, whilst in another study women with $\mathrm{PBC}$ were younger at first pregnancy than controls. A role for xenobiotics was identified. Hair dyes and nail polish may be risk factors. A study from Japan showed higher risk in atomic bomb survivors. The reviewer concluded that investigations in mouse models could unravel relevant gene-environment interactions.

Finally, Smyk and colleagues review both genetic and environmental agents (Smyk D, Rigopoulou EI and Bogdanos DP. PBC triggers in water reservoirs, coal mining areas and waste disposal sites: from Newcastle to New York). Evidence for the role of environmental factors is supported by migration studies. A role for both genetic and environmental factors is supported by both familial and non-familial clustering. Putative environmental factors reviewed included contaminated water, infections and xenobiotics. They suggested that the gram-negative bacterium Novosphingobium aromaticivorans may be implicated, which was interesting because it is capable of metabolizing oestrogens and it can induce PBC-specific anti-mitochondrial antibodies in immunized mice. They were optimistic that, although many agents may be implicated in some way, there should be more conclusive evidence regarding aetiology in the near future.

In conclusion, all of the reviewers were agreed that aetiology is far from straight forward and far from clear. They concur that future efforts should involve the integration of separate investigative strands into a fully comprehensive model. In particular gene-environment interactions should be studied in greater detail.

\section{Reference}

[1] R.J. McNally, S. Ducker and O.F. James, Are transient environmental agents involved in the cause of primary biliary cirrhosis? Evidence from space-time clustering analysis, Hepatology $\mathbf{5 0}$ (2009), 1169-1174. 


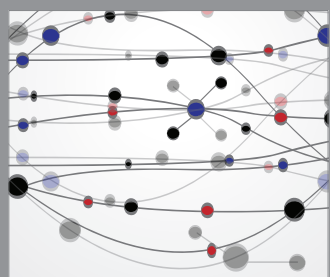

The Scientific World Journal
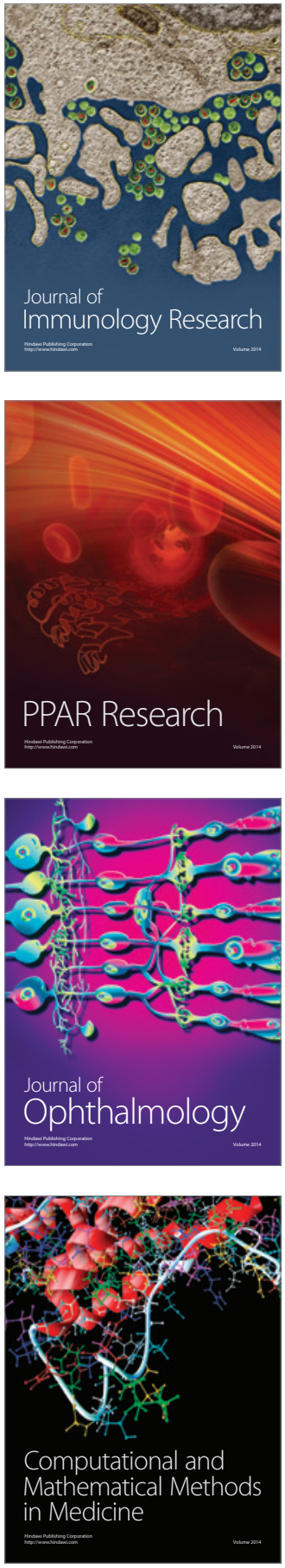

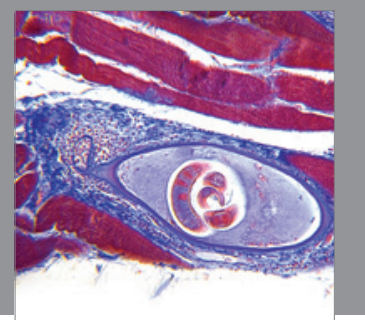

Gastroenterology

Research and Practice
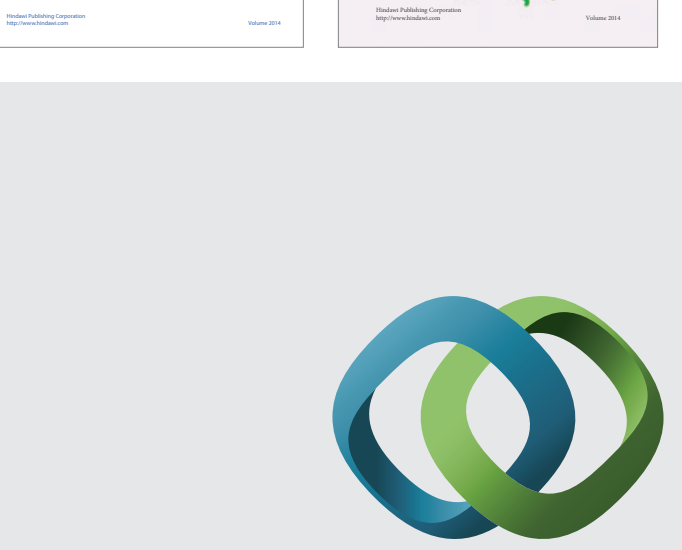

\section{Hindawi}

Submit your manuscripts at

http://www.hindawi.com
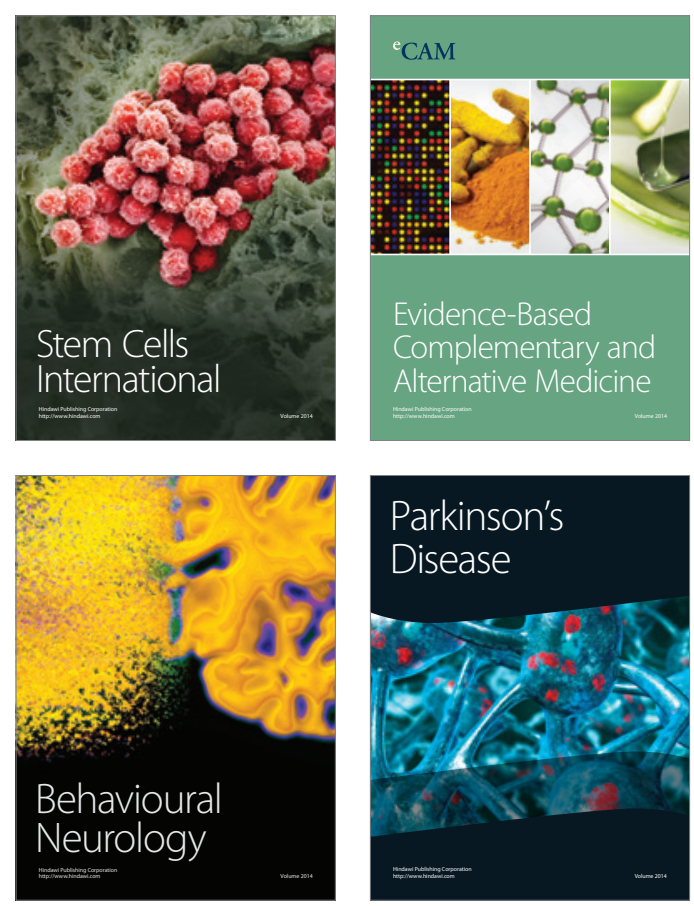

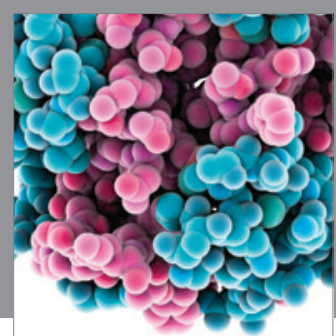

Journal of
Diabetes Research

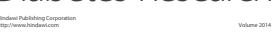

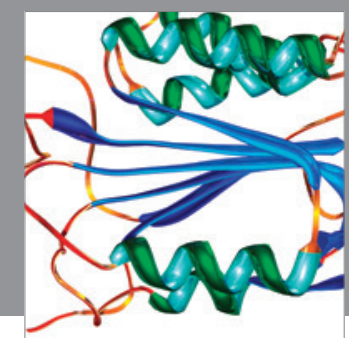

Disease Markers
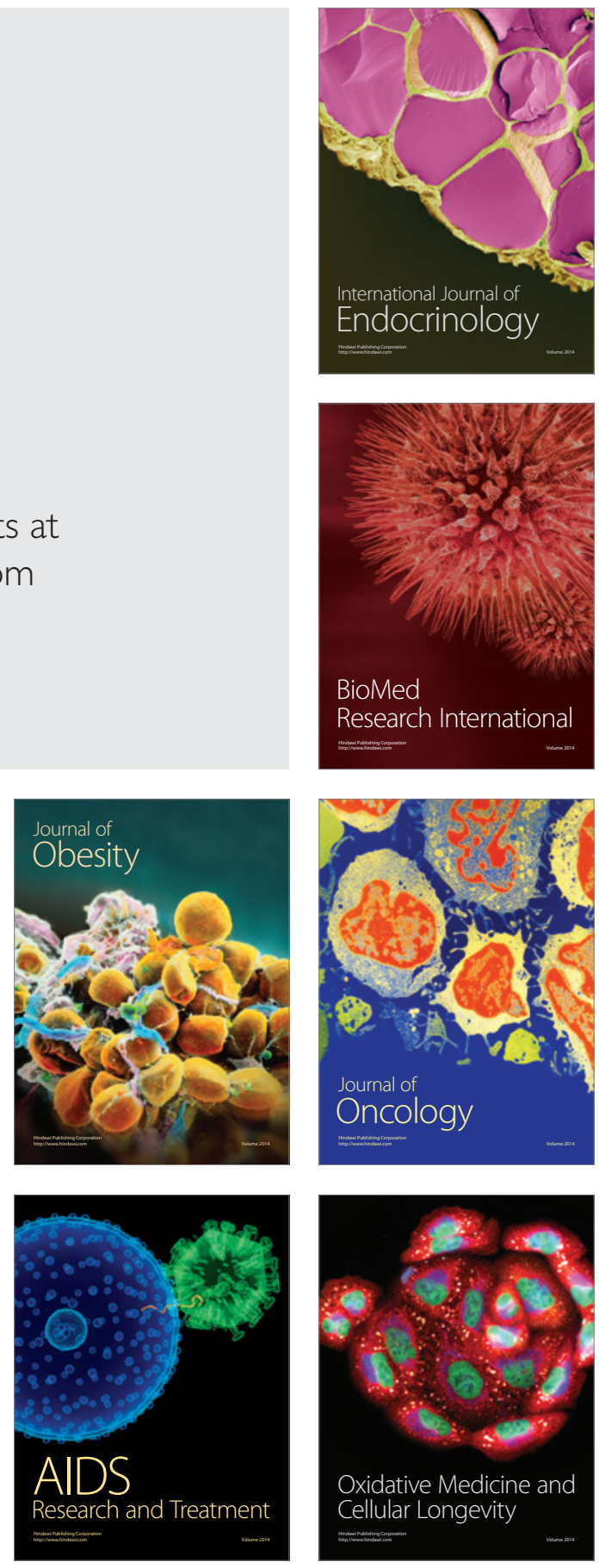